\title{
Boehringer Sandra (préface de D. M. Halperin), L'homosexualité féminine dans l'Antiquité grecque et romaine
}

Paris, Les Belles Lettres, 2007, 405 pages.

Claudine Leduc

\section{CpenEdition}

\section{Journals}

\section{Édition électronique}

URL : https://journals.openedition.org/clio/9052

DOI : 10.4000/clio.9052

ISSN : 1777-5299

\section{Éditeur}

Belin

\section{Édition imprimée}

Date de publication : 15 décembre 2008

Pagination : 275-307

ISSN : 1252-7017

Référence électronique

Claudine Leduc, « Boehringer Sandra (préface de D. M. Halperin), L'homosexualité féminine dans l'Antiquité grecque et romaine », Clio. Histoire, femmes et sociétés [En ligne], 28 | 2008, mis en ligne le 16 décembre 2008, consulté le 22 avril 2022. URL : http://journals.openedition.org/clio/9052 ; DOI : https://doi.org/10.4000/clio.9052

Ce document a été généré automatiquement le 22 avril 2022.

Tous droits réservés 


\title{
Boehringer Sandra (préface de D. M. Halperin), L'homosexualité féminine dans l'Antiquité grecque et romaine
}

Paris, Les Belles Lettres, 2007, 405 pages.

\author{
Claudine Leduc
}

1 Somme sans faille et synthèse brillante, cet ouvrage fera date. L'étude, une première, porte très exactement sur les relations sexuelles entre personnes de sexe féminin (p. 27). Le champ de recherche exploré est impressionnant par son ampleur. Il s'étend presque sur un millénaire ( $\mathrm{vII}^{\mathrm{e}} \mathrm{s}$. av. JC et $\mathrm{III}^{\mathrm{e}} \mathrm{s}$. ap. JC) et embrasse donc des contextes géographiques, sociaux et culturels très différents: la cité grecque archaïque et classique, les royaumes hellénistiques et la Rome impériale. Il a pour limite la conversion du monde gréco-romain au christianisme à partir du $\mathrm{III}^{\mathrm{e}} \mathrm{s}$ (p. 33). Toutes les sociétés concernées par l'enquête relèvent donc d'une tradition religieuse polythéiste dépourvue de code de moralité : leur position vis-à-vis des relations sexuelles entre personnes de même sexe ne relève jamais de l'anathème, mais dépend des conditions historiques, du code social en usage et de la catégorie littéraire à laquelle appartient la source qui en fait état.

2 La problématique de Sandra Boehringer est « constructionniste ». Plus que de sexualité, elle préfère parler de pratiques sexuelles, car, dit-elle, dans l'Antiquité, un individu n'a pas de sexualité. Il se livre aux aphrodisia ou aux veneria, aux choses de la jouissance sexuelle et a (ou n'a pas) à leur égard de "diététique ", de maîtrise de soi (p. 29). À la différence du sexe qui est naturel, ces pratiques sont une construction sociale et culturelle, inscrite dans le temps et l'espace, et non un objet transhistorique et transgéographique, un invariant (p. 21). L'enquête de Sandra Boehringer s'inscrit donc en France dans la lignée des travaux pionniers : M. Foucault et son Histoire de la sexualité dès 1976 ; l'ouvrage de K. J. Dover, Greek Homosexuality (1978) traduit en 1982 ; l'article de P. Veyne, «L'homosexualité à Rome ", publié en 1981 dans le numéro 30 de L'Histoire. Elle est aussi immergée, ainsi qu'en témoigne une très imposante bibliographie, dans le puissant courant de recherche nord-américain impulsé, depuis les années 1980, par les 
mouvements féministe, queer, gay et lesbien. Ses références théoriques en appellent en particulier à deux auteurs (accessibles en français !) : J. J. Winkler (Désir et contraintes en Grèce ancienne, Paris, 2005, traduction Sandra Boehringer et N. Picard) et D. M. Halperin (Cent ans d'homosexualité et autres essais sur l'amour grec, Paris, 2000), qui dans la préface de l'ouvrage manifeste sa communauté de point de vue avec l'auteure.

Bien que daté de 2007 et inscrit dans une longue lignée scientifique, l'ouvrage de Sandra Boehringer est pionnier en son domaine. Alors que l'homosexualité masculine dans l'Antiquité grecque fait l'objet, depuis une trentaine d'années, de nombreuses publications, l'homosexualité féminine n'a pas suscité de recherche particulière $\mathrm{y}$ compris en langue anglaise. Il n'y a alors en français que deux publications qui en aborde l'étude, Les choeurs de jeunes filles en Grèce ancienne (1977) de C. Calame et un article de N. Ernoult sur «L'homosexualité féminine chez Platon» dans la Revue française de psychanalyse I (1994). Pourquoi un tel décalage dans la recherche, y compris aux U.S.A, entre homosexualité masculine et homosexualité féminine? K.J. Dover l'explique par l'état de la documentation, pléthorique dans un cas et fort mince dans l'autre, mais il ne s'interroge pas sur la signification du déséquilibre. Les occurrences sur l'homosexualité féminine sont non seulement peu nombreuses, mais elles sont brèves, souvent fragmentaires, et toujours éclatées dans des sources dispersées et de statuts très différents. Au problème de leur rareté s'ajoute donc celui de l'extrême difficulté de leur exploitation. La progression d'une enquête pleine d'embûches exigeait de Sandra Boehringer d'associer à la rigueur, prudente et minutieuse, d'une philologue très exigeante, la passion d'une chercheuse profondément investie dans son sujet.

Les sources sur l'homosexualité féminine n'offrent pratiquement qu'un seul type de documents - des occurrences textuelles - ce qui interdit le procédé de croisement préconisé par les historiens de l'Antiquité entre littérature, épigraphie et iconographie. Alors qu'en pays grec, les images de la céramique à figures noires et rouges sont essentielles à l'étude de l'homosexualité masculine, comme en témoigne l'ouvrage de K.J. Dover, elles sont pratiquement silencieuses sur les relations sexuelles entre femmes (p. 145). S. Boehringer en réduit encore l'inventaire. C'est une erreur, dit-elle, d'interpréter le godemiché, que des femmes portent sur certains vases, comme un instrument de lesbienne et le signe marqueur d'une scène d'homosexualité féminine (p. 146). Il suffit de lire la Lysistrata d'Aristophane pour se rendre compte qu'il est d'un usage très étendu. Il y aurait donc au maximum 5 représentations figurées susceptibles de faire référence à des amours féminines et toutes se prêtent à des interprétations contradictoires: quand une femme est représentée touchant le sexe d'une autre femme, s'agit-il d'un rapport sexuel entre personne d'un même sexe ou d'une scène d'épilation? Le silence des images procède certes de "tabous iconiques» (p. 144). La société entend être vue comme un monde qui ignore aussi bien les pratiques de l'homosexualité féminine que certaines pratiques de l'homosexualité masculine (par convention n'est représenté sur les images que le coït intercrural pratiqué par l'éraste). Mais ce silence devient significatif dès qu'est prise en compte la catégorie de vaisselle qui porte en pays grec des représentations érotiques. Il s'agit de la vaisselle de "banquet", donc d'une vaisselle à usage festif exclusivement masculin. Pour l'iconographie, l'image des amours féminines n'est pas un motif érotique susceptible de stimuler le désir des convives (p. 156). Mais l'image du sexe féminin est aussi absente sur les vases et les coupes à boire. Seule la représentation du sexe masculin est 
excitante pour les convives. Les silences de la documentation sont parfois plus révélateurs que de longs développements.

5 «Le voyage dans le passé » de Sandra Boehringer est donc un voyage à travers les textes. Sa longueur et l'hétérogénéité des sources l'ont fait décider de sa démarche: cette dernière «se doit de tenir compte de la chronologie et elle se doit également de consacrer un développement important à la contextualisation de chaque document, document qui est souvent, pour certaines périodes de l'histoire au milieu de décennies de silence » (p. 33). Ce cheminement pas à pas, avec arrêt prolongé sur le locuteur, le code social qui l'environne, le statut de la source où il s'exprime et la finalité de son discours, ralentit sans doute la démonstration par sa grande minutie, mais il interdit à l'auteure de s'enfermer dans la restriction du champ qu'imposerait sa subjectivité (p. 26), le danger qui guette toute recherche. Chronologique, cette approche « se révèle être simultanément une approche par genres littéraires : elle fait apparaitre qu'il y a un genre pour chaque époque et une époque pour chaque genre» ( $p .33)$. Les relations sexuelles entre femmes sont évoquées dans des poèmes lyriques à l'époque archaïque, dans des oeuvres philosophiques à l'époque classique et, pour beaucoup, dans la satire à l'époque romaine.

Comme tout historien, Sandra Boehringer aborde l'étude du passé à partir du présent, mais elle le fait en toute conscience des effets, à la fois dynamiques et destructeurs, de l'anachronisme dans une réflexion (p.26), qui fait suite à celle de N. Loraux dans "Éloge de l'anachronisme en histoire»: «toutes nos questions au passé sont anachroniques (même lorsqu'on pense qu'elles ne le sont pas), lestées qu'elles sont des connotations actuelles et de strates de discours qui se sont accumulées ». Toutes nos catégories sont anachroniques: l'historien-anthropologue doit apprendre la langue de l'autochtone (qu'il connaît à peine quoique helléniste !) et « désapprendre » la sienne. En quelques lignes, Sandra Boehringer met en évidence la distorsion qu'introduirait le placage de nos grilles analytiques sur les représentations des pratiques sexuelles de l'Antiquité (p. 30) : « Les anciens n'ont jamais élaboré ni pensé une catégorie homogène qui engloberait indistinctement hommes et femmes de tous milieux sociaux ayant pour unique caractéristique commune d'être attirés par les personnes du même sexe qu'eux - pas plus qu'ils ne lui ont opposé une catégorie qui engloberait hommes et femmes attirés par les personnes de l'autre sexe ». Plus encore l'analyse des sources grecques et romaines a montré qu'il n'existe pas de catégories communes aux homosexuels et aux homosexuelles, mais aussi qu'il n'existe pas de catégorie « homosexualité masculine »... et de catégorie "homosexualité féminine ». Pour que de telles catégories existent, il faut que l'identité de sexe soit perçue comme une caractéristique signifiante ; or, dans le monde antique, un individu, homme ou femme, se définit d'abord par son statut politique et social. C'est donc en montrant l'absence de concordance des sources avec notre catégorie "homosexualité féminine » que Sandra Boehringer fait apparaitre le positionnement propre au monde gréco-romain sur les relations sexuelles entre femmes : elles ne sont pas l'objet d'une évaluation en soi, mais sont perçues en fonction du code social en usage aux époques concernées, un code qui fait prendre en compte leur statut, leur rang, les modalités de leur relation, la maittrise de leurs passions. L'application de cette méthode à l'étude de sources aussi différentes que les poèmes d'Alcman et de Sappho, le Banquet et les Lois de Platon, les Métamorphoses d'Ovide ou les satires de Martial permet à S. Boehringer d'échapper aux problématiques 
traditionnelles, aux lectures hâtives et aux clichés sempiternels et de présenter, dans chaque cas, une étude aussi profonde qu'originale. 